\title{
Surface Functionalization of ZnO Nanotetrapods with Photoactive and Electroactive Organic Monolayers
}

\author{
Dongfang Liu, Wei Wu, Yongfu Qiu, Shihe Yang* \\ Department of Chemistry, The Hong Kong University of Science and Technology, Clear Water \\ Bay, Kowloon, Hong Kong, China \\ Si Xiao, Qu-Quan Wang \\ Department of Physics and Key Laboratory of Acoustic and Photonic Materials and Devices of \\ Ministry of Education, Wuhan University, Wuhan 430072, China \\ Lu Ding, Jiannong Wang \\ Department of Physics, The Hong Kong University of Science and Technology, Clear Water Bay, \\ Kowloon, Hong Kong, China
}

\section{Supporting Information}




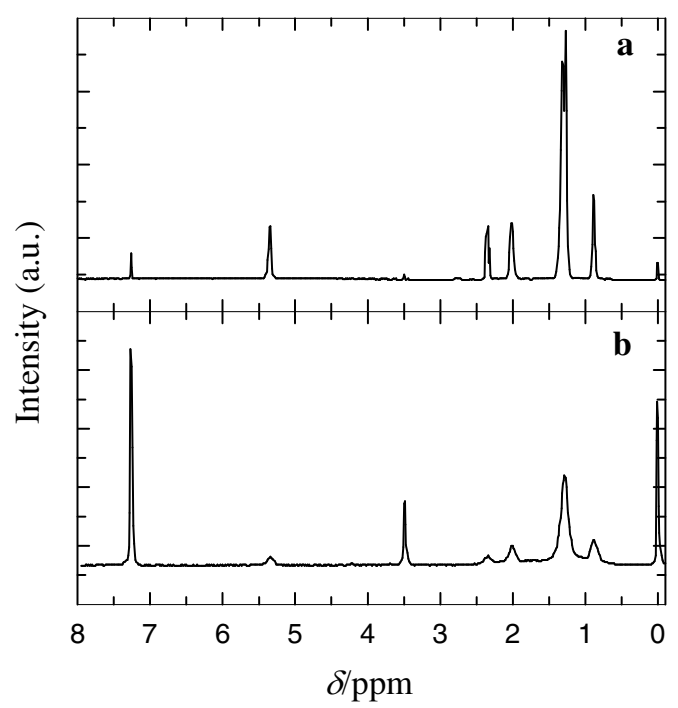

Figure S1. $\quad{ }^{1}$ H NMR spectra of free oleic acid (a) and nanotetrapods $\mathbf{1}$ (b).

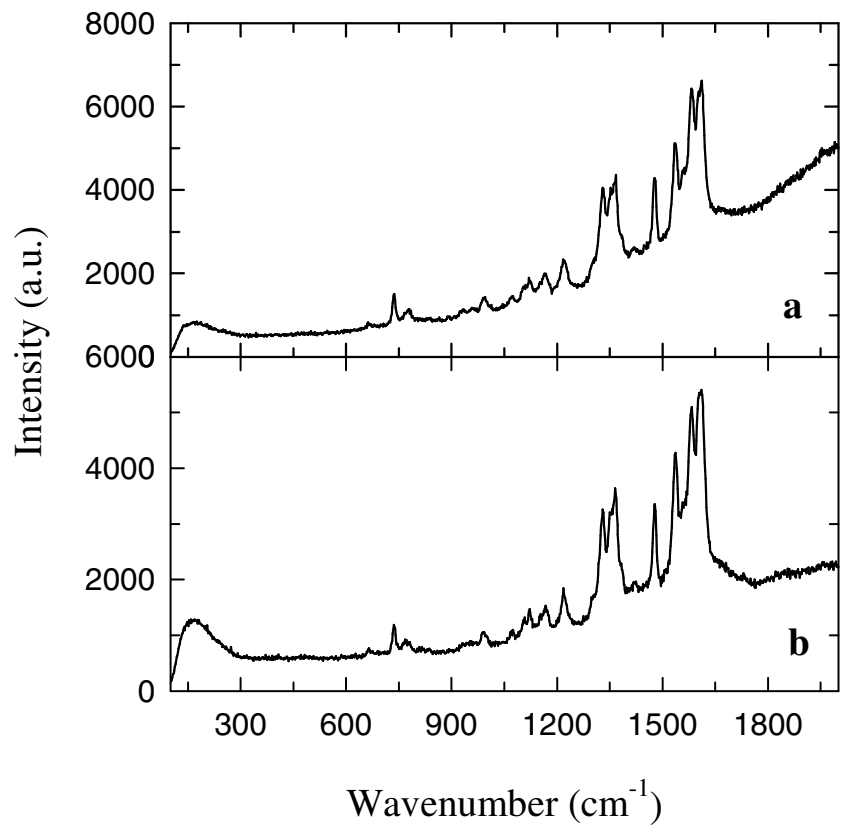

Figure S2. Raman spectra of free protoporphyrin IX disodiam salt (a) and nanotetrapods 2 (b). Both were measured using solid samples. 


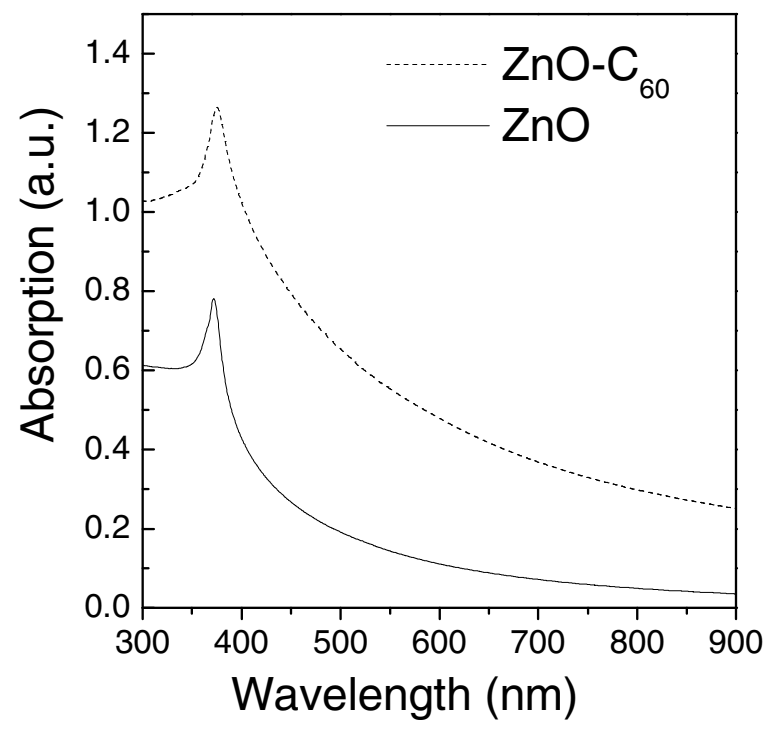

Figure S3. Uv-Vis spectra of $\mathrm{ZnO}$ nanotetrapod (solid line) and $\mathrm{ZnO}-\mathrm{C}_{60}$ nanotetrapod 5 (dot line).

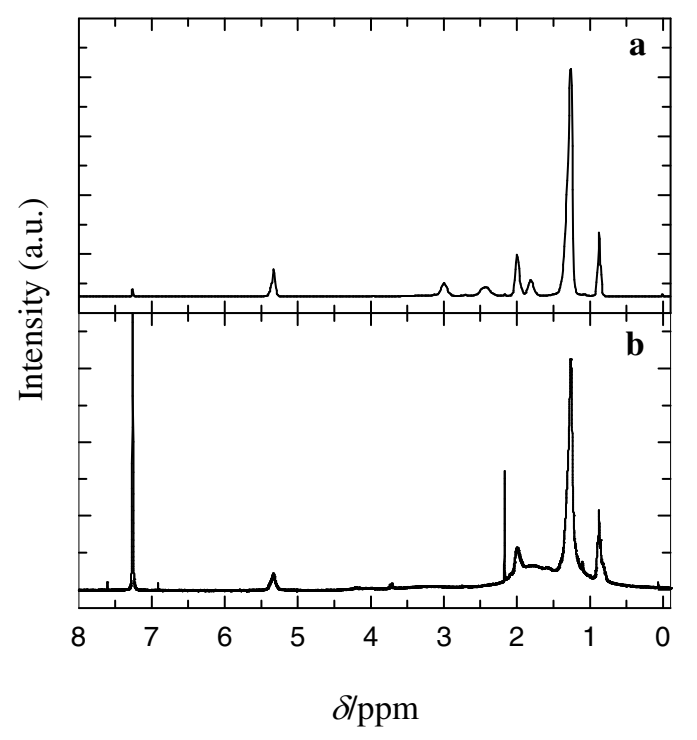

Figure S4. $\quad{ }^{1}$ H NMR spectra of free phosphonic acid 7 (a) and nanotetrapods 8 (b). 


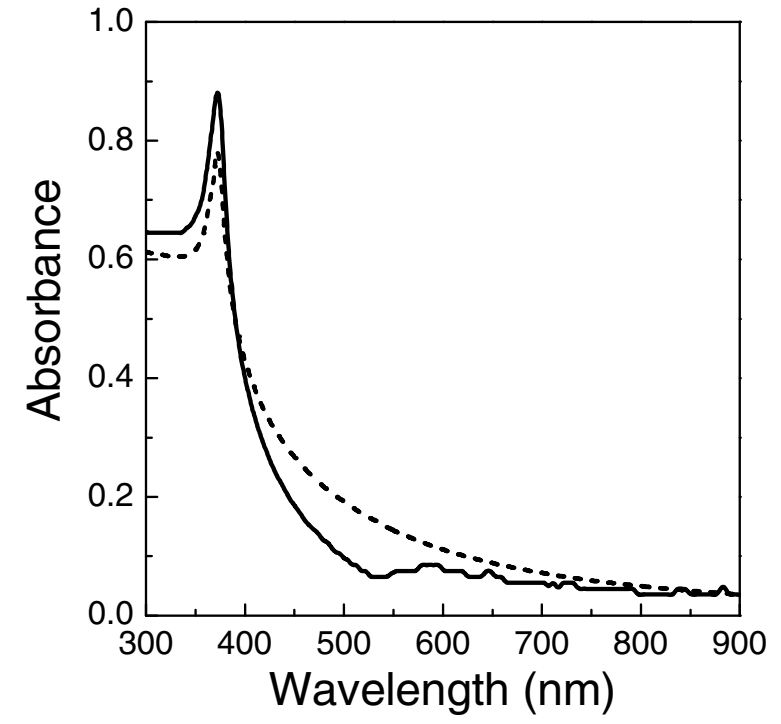

Figure S5. UV-vis absorption spectrum of nanotetrapods $\mathbf{8}$ in chloroform.

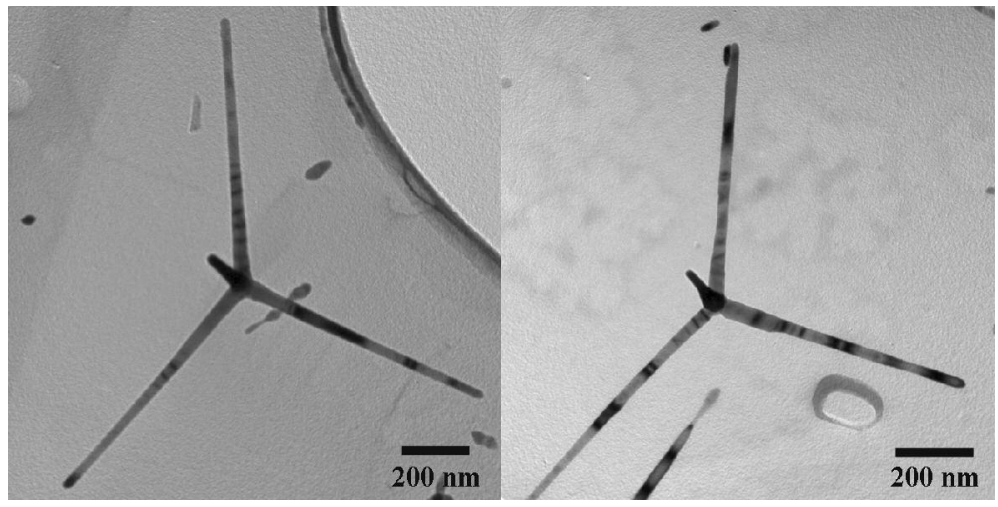

Figure S6. TEM images of nanotetrapods 8 . 


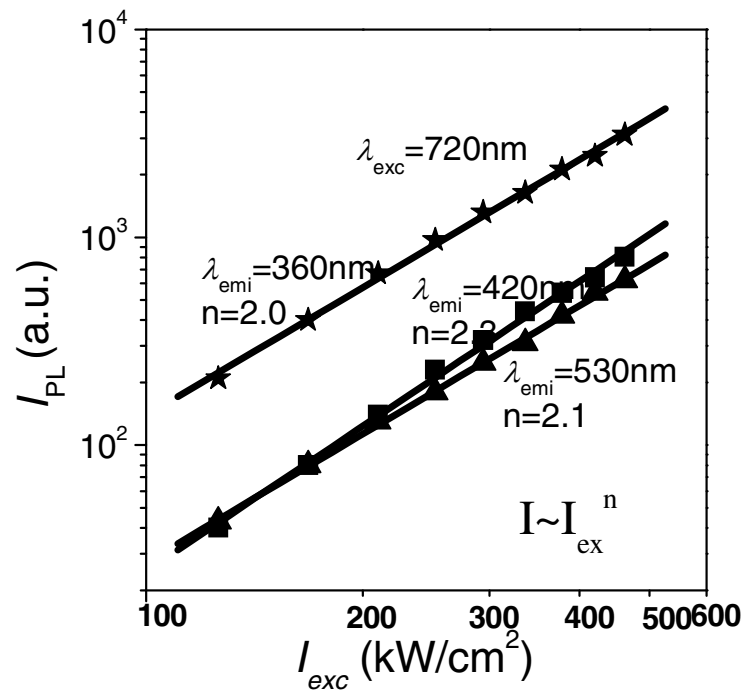

Figure S7. PL peak value of nanotetrapods 2 at 360, 420, and $546 \mathrm{~nm}$ vs. excitation intensity.

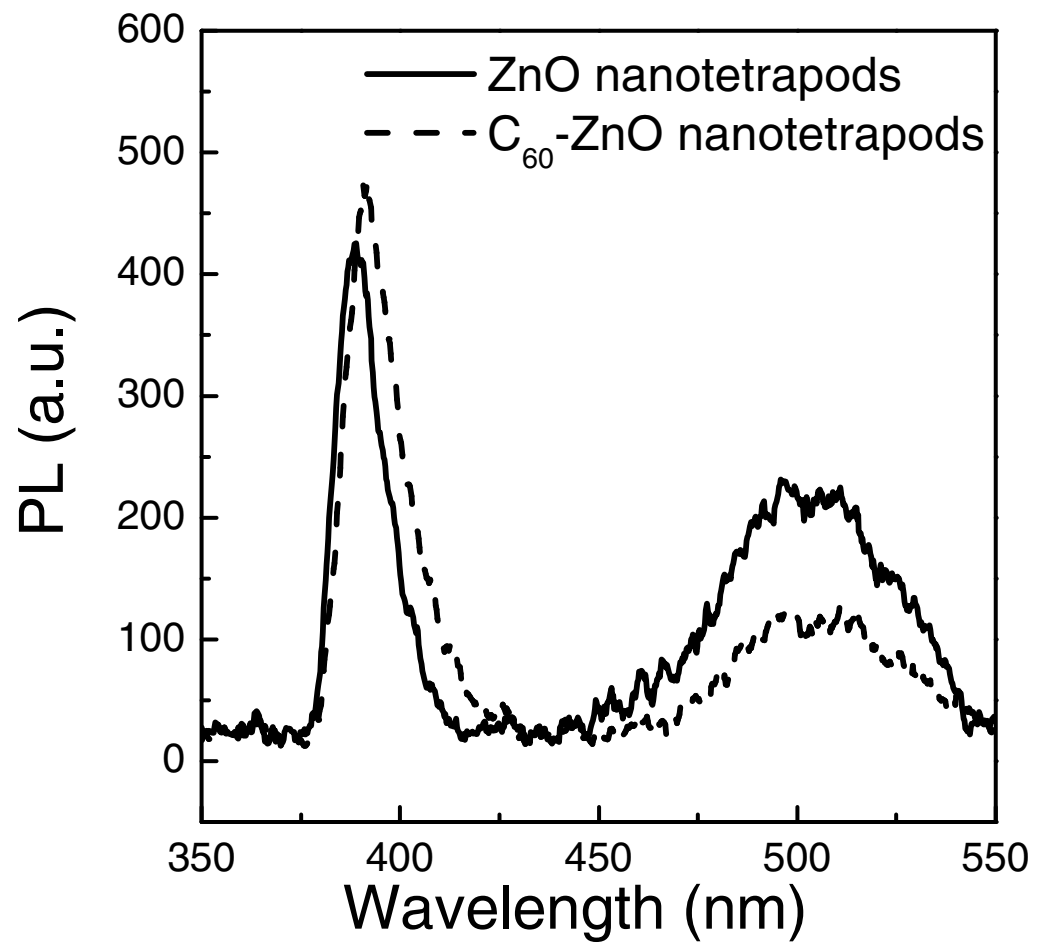

Figure S8. Typical two-photon luminescence spectra of the $\mathrm{C}_{60}-\mathrm{ZnO}$ (solid line) and $\mathrm{ZnO}$ nanotetrapods (dotted line). 